\title{
Silicon Nitride Photonics for the Near-Infrared
}

\author{
Thalía Domínguez Bucio, Cosimo Lacava, Marco Clementi, Joaquin Faneca, Ilias Skandalos, Matteo Galli, \\ Kapil Debnath, Anna Baldycheva, Periklis Petropoulos, Frederic Gardes.
}

(Invited Paper)

\begin{abstract}
In recent years, silicon nitride has drawn attention for the realisation of integrated photonic devices due to its fabrication flexibility and advantageous intrinsic properties that can be tailored to fulfill the requirements of different linear and non-linear photonic applications. This paper focuses on our progress in the demonstration of enhanced functionalities in the near infrared wavelength regime with our low temperature $\left(<350^{\circ} \mathrm{C}\right) \mathrm{SiN}$ platform. It discusses (de)multiplexing devices, nonlinear all optical conversion, photonic crystal structures, the integration with novel phase change materials, and introduces applications in the $2 \mu \mathrm{m}$ wavelength range.
\end{abstract}

Index Terms-Photonics, Silicon Nitride, (De)Multiplexers, Nonlinear, Photonic Crystals, Phase Change Materials.

\section{INTRODUCTION}

$\mathbf{T}$ He success of silicon photonic devices has gone beyond data interconnects and has undoubtedly progressed to a wide range of applications such as sensing, all optical processing and quantum photonics [1]-[3]. However, some of the intrinsic properties of silicon pose challenges to the successful demonstration of devices for these new applications. For instance, although beneficial for the realization of switching functionalities, the high thermo-optic coefficient of silicon makes SOI devices strongly sensitive to temperature variations. In addition, the presence of two-photon absorption deems SOI devices potentially inefficient for high power density and all-optical processing applications. As a result, there is an increasing interest in exploring flexible CMOS compatible materials with optical properties and refractive indices in between those of silicon and $\mathrm{SiO}_{2}$ that can be introduced in photonic integrated circuits to complement the functionality and overcome the limitations of silicon. Some of the materials that have been considered for the near-infrared include hydrogenated amorphous silicon [4]-[6], silicon oxynitride [7]-[9] and silicon nitride [10]-[12].

The authors acknowledge the financial support of the EU 2020 project "COSMICC" and the Engineering and Physical Sciences Research Council (EPSRC) with the grants "Electronic-Photonic Convergence" (EP/N03247/1), "Silicon Photonics for Future Systems" (EP/L00044X/1), "CORNERSTONE" (EP/L021129/1) and "Rockley Photonics and the University of Southampton: A Prosperity Partnership" (EP/R003076/1). (Corresponding author: T. Domínguez Bucio)

T. Domínguez Bucio, C. Lacava, I. Skandalos, P. Petropoulos and F. Gardes are with the Optoelectronics Research Centre, University of Southampton, Highfield, Southampton SO17 1BJ, UK. (e-mail: T.Dominguez_Bucio@soton.ac.uk)

M. Clementi and M. Galli are with the Dipartimento di Fisica, Università degli Studi di Pavia, via Agostino Bassi 6, 27100 Pavia, Italy.

J. Faneca and A. Baldycheva is with the EPSRC centre for doctoral training, University of Exeter, Exeter, UK.

K. Debnath is with the Department of Electronics and Electrical Communication Engineering, Indian Institute of Technology, Kharagpur, West Bengal 721302, India
Among these materials, silicon nitride (SiN) has gained significant attention not only due to its CMOS compatibility but also due to its flexible optical properties, that can be easily tuned during fabrication to fulfill the specific requirements of different applications. SiN has a wide optical band-gap that can be varied from 2.7 to $5.0 \mathrm{eV}$ by increasing the $\mathrm{N} / \mathrm{Si}$ ratio of the material. This widely tunable band-gap sets the bottom limit of the transparency window of $\mathrm{SiN}$ between 250 and $400 \mathrm{~nm}$ in the lower end of the visible spectrum, while the top limit extends all the way to the mid-infrared [13], [14]. Similarly, the refractive index of SiN can be tuned between 1.7 and 3.0 by reducing the N/Si ratio of the material. Even though the refractive index of $\mathrm{SiN}$ is lower than that of silicon, it provides a good optical confinement ( $60-80 \%)$ with the advantage of having a higher tolerance to surface roughness and dimensional variations in the near-infrared [15], [16]. The thermo-optic coefficient of $\operatorname{SiN}\left(10^{-5}{ }^{\circ} \mathrm{C}^{-1}\right)$ is one order of magnitude lower than that of silicon resulting spectral variations as small as $11 \mathrm{pm} /{ }^{\circ} \mathrm{C}$ under variable temperature conditions [11], [17]. Finally, SiN exhibits negligible twophoton absorption [18] at $1550 \mathrm{~nm}$. All these features have made $\mathrm{SiN}$ an ideal candidate for the realization of a variety of photonic devices for linear and non-linear applications covering visible to mid-infrared wavelengths.

In this paper, we present our progress in the demonstration of enhanced linear and nonlinear functionalities in the near infrared wavelength regime with our low temperature $\left(<350{ }^{\circ} \mathrm{C}\right)$ SiN platform with refractive indices between 1.5 and 2.7 [19]. We start by describing the linear and nonlinear properties of the platform. Afterwards, we discuss the use of the platform to demonstrate (de)multiplexing in the $\mathrm{O}$ band wavelength window, nonlinear all optical wavelength conversion and photonic crystal structures with enhanced nonlinear properties in the $\mathrm{C}$ band. We finalize by introducing the integration of $\mathrm{SiN}$ structures with novel phase change and 2D materials, along with the use of the platform for the $2 \mu \mathrm{m}$ wavelength range.

\section{LineAR AND NONLINEAR OptiCAL PROPERTIES}

Silicon nitride is commonly used in the form of hydrogenated amorphous films. The composition of these films tends to depart from the stoichiometric $\mathrm{N} / \mathrm{Si}$ ratio of 1.3 and is characterized by the presence of $\mathrm{Si}-\mathrm{H}$ and $\mathrm{N}-\mathrm{H}$ bonds [20], [21]. These hydrogen bonds are particularly problematic in the near-infrared as they act as absorption centres that lead to undesirable losses [21]. In fact, $\mathrm{N}-\mathrm{H}$ bonds generate an absorption peak close to $1550 \mathrm{~nm}$ within the $\mathrm{C}$ wavelength band [10]. As a result, $\mathrm{SiN}$ films are typically grown using high temperature processes $\left(>1000^{\circ} \mathrm{C}\right)$, such as low pressure 
chemical vapour deposition (LPCVD), that have the sufficient energy to break the hydrogen bonds and, so, enable the realization of devices with losses $<1.0 \mathrm{~dB} / \mathrm{cm}$ [22], [23]. However, the increasing interest in multilayer integration has led to the study of deposition methods that can provide a comparable material quality with temperatures compatible with CMOS back-end-of-line integration $\left(<400^{\circ} \mathrm{C}\right)$, such as plasma enhanced chemical vapour deposition (PECVD) and hot-wire chemical vapour deposition (HWCVD) [24]-[26].

One alternative that has been explored to reduce the hydrogen available during the PECVD deposition process is a modified recipe that uses $\mathrm{N}_{2}$ as a precursor gas instead of $\mathrm{NH}_{3}$. We have have exploited this approach to achieve propagation losses $<1 \mathrm{~dB} / \mathrm{cm}$ in the O-band $(\lambda=1310 \mathrm{~nm})$ and $\sim 1.2 \mathrm{~dB} / \mathrm{cm}$ in the C-band $(\lambda=1550 \mathrm{~nm})$ for different $\mathrm{N} / \mathrm{Si}$ concentrations with a maximum processing temperature of $350^{\circ} \mathrm{C}$ [19]. This section discusses how the deposition parameters can be modified to tune the linear and non-linear optical properties of $\mathrm{NH}_{3}$-free PECVD SiN films.

\section{A. Tunable Linear Optical Properties}
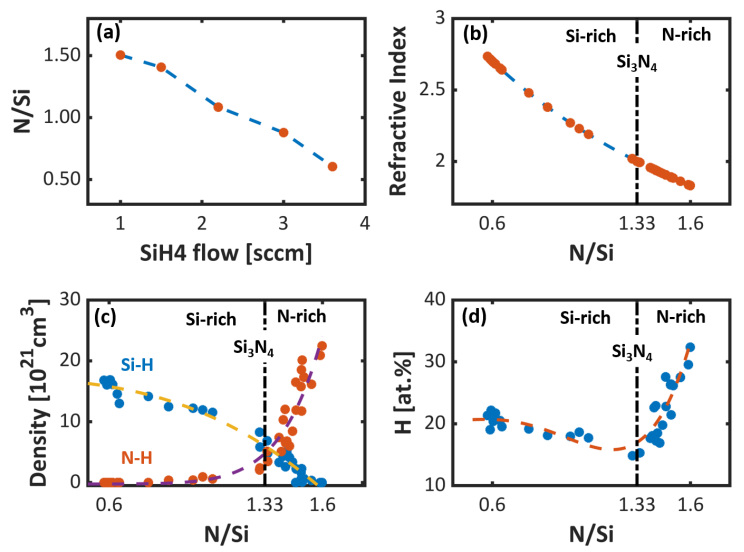

Fig. 1. (a) N/Si ratio vs $\mathrm{SiH}_{4}$ flow, (b) Refractive index $(\lambda=632 \mathrm{~nm})$, (c) $\mathrm{Si}-\mathrm{H}$ and N-H, and (d) Hydrogen concentration as a function of N/Si. [19].

Reference [19] discusses in detail the experiments and characterisation techniques used to evaluate the effect of the deposition parameters on the optical properties of $\mathrm{NH}_{3}-$ free PECVD SiN. The experiments show that most optical properties reflect mainly the changes exerted by the deposition conditions on the stoichiometry of the films. As a result, they are predominantly affected by the $\mathrm{SiH}_{4}$ flow, which is the deposition parameter that has the greatest effect on their N/Si ratio. Fig. 1 (a) shows that using this parameter the N/Si ratio of the films can be tuned between 0.6 and 1.6 to obtain either N-rich $(>1.3)$, Si-rich $(<1.3)$ or stoichiometric $(\sim 1.3)$ compositions. In a similar manner, Fig. 1 (b) shows that the refractive index varies between 1.8 for $\mathrm{N}$-rich layers and 2.6 for Si-rich layers within the tuning range of their N/Si ratio. As illustrated in Fig. 1 (c) and (d), the bond configuration of the films is also intrinsically related to their N/Si ratio. Si-rich and N-rich films tend to have a high concentration of $\mathrm{Si}-\mathrm{H}$ and $\mathrm{N}-\mathrm{H}$ bonds, respectively, that are responsible for undesirable absorption losses in the 1510-1650 $\mathrm{nm}$ ange [10]. In addition, Si-rich films have a high concentration of $\mathrm{Si}-\mathrm{Si}$ bonds responsible for defects that can cause additional losses. On the other hand, stoichiometric films exhibit a minimal hydrogen incorporation ( $15 \%$ ) compared to standard PECVD $\mathrm{SiN}(>20 \%)$ [24].
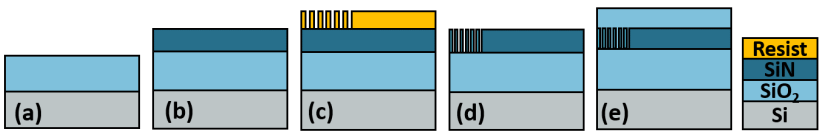

Fig. 2. Device fabrication process: (a) silicon substrate with $2-3 \mu \mathrm{m}$ of thermal $\mathrm{SiO}_{2}$, (b) PECVD SiN deposition, (c) Lithography, (d) SiN ICP etching with $\mathrm{SF}_{6}: \mathrm{CHF}_{3}$, and (e) $1 \mu \mathrm{m} \mathrm{PECVD} \mathrm{SiO}_{2}$ deposition.

Waveguides with widths between 900-1200 nm and lengths up to $1.7 \mathrm{~cm}$ were fabricated on $300 \mathrm{~nm}$ thick SiN films following the process detailed in Fig. 2 to investigate the effect of material properties on the linear optical losses $(\alpha)$ of the films in the $\mathrm{O}(\lambda=1310 \mathrm{~nm})$ and $\mathrm{C}(\lambda=1550 \mathrm{~nm})$ using cut-back measurements. The results indicated that the losses at both wavelengths are highly correlated with the N/Si ratio of the $\mathrm{SiN}$ films. Fig. 3 shows that propagation losses at $1310 \mathrm{~nm}$ decrease to $<1.0 \mathrm{~dB} / \mathrm{cm}$ with increasing $\mathrm{N} / \mathrm{Si}$ ratio and, so, $\mathrm{N}$-rich layers exhibit lower propagation losses in the O-band. This suggests that losses at $1310 \mathrm{~nm}$ are limited by the Rayleigh scattering inherent to the structure of the materials. Hence, since Si-rich films tend to have more $\mathrm{Si}-\mathrm{H}$ and $\mathrm{Si}-\mathrm{Si}$ bond defects, they present higher losses in the Oband. On the other hand, propagation losses at $1550 \mathrm{~nm}$ reach a minimum value of $1.2 \mathrm{~dB} / \mathrm{cm}$ close to the stoichiometric ratio $\mathrm{N} / \mathrm{Si}=1.33$ following the hydrogen concentration of the films indicating that they are primarily the result of the absorption induced by the presence of N-H bonds. As a result, Si-rich films exhibit lower losses in the C-band due to the absence of $\mathrm{N}-\mathrm{H}$ bonds in their structure, while N-rich layers have increased absorption losses.

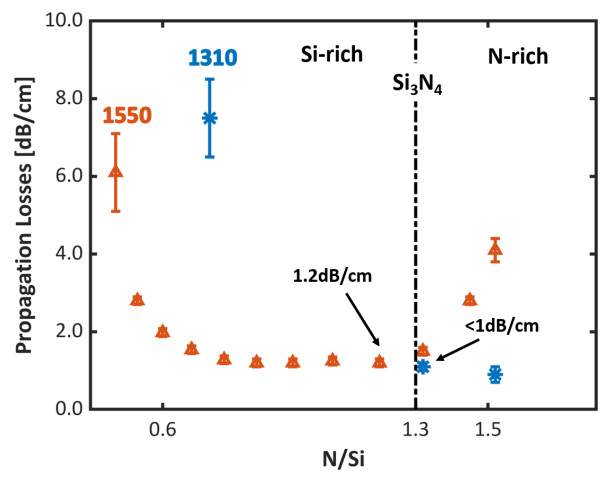

Fig. 3. Propagation losses measured for waveguides fabricated $\mathrm{onNH}_{3}$-free PECVD films with different N/Si ratios [19].

As show in table I, the losses at $1550 \mathrm{~nm}$ tend to be higher than at $1310 \mathrm{~nm}$ due to the presence of $\mathrm{N}-\mathrm{H}$ bonds in the SiN films regardless of the deposition method. Nevertheless, the losses achieved with $\mathrm{NH}_{3}$-free $\mathrm{SiN}$ are lower than the best demonstrated with standard PECVD. On the contrary, the propagation losses at $1310 \mathrm{~nm}$ are only comparable to those 
of standard PECVD. This result demonstrates that the $\mathrm{NH}_{3}$ free approach considerably reduces the propagation losses of the PECVD SiN layers without high temperature processing. In fact, losses $\sim 1.2 \mathrm{~dB} / \mathrm{cm}$ can be achieved at $1550 \mathrm{~nm}$ with stoichiometric films that have minimal hydrogen incorporation.

TABLE I

Propagation Losses of SiN WAVEguides. *THIS WORK.

\begin{tabular}{cccc}
\hline Material & $\begin{array}{c}\text { Core } \\
{\left[\mathbf{n m}^{\mathbf{2}}\right]}\end{array}$ & $\begin{array}{c}\text { Loss@ 1550 } \\
{[\mathbf{d B} / \mathbf{c m}]}\end{array}$ & $\begin{array}{c}\text { Loss@ 1310 } \\
{[\mathbf{d B} / \mathbf{c m}]}\end{array}$ \\
\hline LPCVD & $1000 \times 400$ & $0.72[22]$ & $0.24[23]$ \\
PECVD & $1000 \times 400$ & $3.00[27]$ & $0.85[27]$ \\
HWCVD & $1000 \times 300$ & $6.1[26]$ & $5.7[26]$ \\
$\mathrm{NH}_{3}$-free PECVD & $700 \times 400$ & $2.10[10]$ & - \\
& $1000 \times 300$ & $1.20 *$ & $1.00 *$ \\
\hline
\end{tabular}

\section{B. Tunable Nonlinear Optical Properties}

In the same way that it is possible to significantly tune the linear properties of silicon nitride, it is possible to tune its nonlinear (Kerr response) properties allowing for unprecedented control over the design of Kerr-based all-optical signal processing components [28], [29]. As shown in Section II-A, by increasing the quantity of $\mathrm{SiH}_{4}$ during the deposition process of the material it is possible to achieve Si-rich $\mathrm{SiN}$ layers. Here, we report a comprehensive experimental measurement campaign, showing both the linear and nonlinear performance of three different $\mathrm{Si}$-rich silicon nitride material compositions. The basic properties of the three layers, deposited on a thermal $\mathrm{SiO}_{2}$ substrate, are shown in Tab. II. A set of fully-etched waveguides with different widths (ranging from $0.5-1.5 \mu \mathrm{m}$ ) were patterned onto the three layers and used for the experimental measurements. Grating- (for low power measurements) and tapered butt- (for high power measurements) couplers were also written to facilitate light coupling from external sources using optical fibres. The first set of measurements aimed at identifying the propagation loss of each structure by measuring the optical transmission power in different length waveguides $(1-5 \mathrm{~cm})$ using the cut-back method. In this set of experiments, coupling between optical fibres and waveguides was realized using grating couplers.

TABLE II

DEPOSITED SI-RICH SILICON NITRIDE LAYER CHARACTERISTICS

\begin{tabular}{ccc}
\hline Layer ID & $\begin{array}{c}\text { Refractive index } \\
\text { (@ 1550 nm) }\end{array}$ & $\begin{array}{c}\text { Thickness } \\
\text { [nm] }\end{array}$ \\
\hline 01 & 2.01 & 300 \\
02 & 2.49 & 297 \\
03 & 2.71 & 308 \\
\hline
\end{tabular}

As presented in Fig. 4, waveguides from Layers 01 and 02, exhibiting the lowest refractive indices, show the typical exponential dependence of loss versus waveguide width, with narrower waveguides experiencing higher losses due to a greater interaction between the optical mode and the sidewalls. The higher refractive index contrast waveguides written onto Layer 03 does not show this behaviour, and we attributed this to the fact that the optical mode is well confined within the waveguide core even at waveguide widths as small as $500 \mathrm{~nm}$, making the light-to-sidewall interactions less prominent. These results highlight that PECVD Si-rich silicon nitride waveguides with propagation losses approaching $1.5 \mathrm{~dB} / \mathrm{cm}$ were realized. Increased propagation losses were measured for waveguides grown on Layer $03(6 \mathrm{~dB} / \mathrm{cm})$, see Fig.4(c)) revealing that an excessive $\mathrm{Si}$ content in the material composition led to reduced material quality. Since the loss results presented in Fig. 4(c) did not show a significant dependence on the waveguide width, we concluded that the majority of the optical losses observed are due to material absorption [28]. It is widely recognized that one of the main drawbacks of silicon-based nonlinear devices is the strong presence of TPA-related effects. These effects limit the use of silicon devices to relatively low power levels, therefore reducing the device nonlinear efficiency. In [28] we have shown that silicon nitride layers can be properly engineered to show a high Kerr nonlinearity response, with no TPA-related effects, allowing waveguides to be operated at Watt-power levels. A systematic characterization of the nonlinear optical properties of the waveguides, fabricated in the three different layer compositions is presented in this section. The nonlinear response (third order nonlinearities) of an optical waveguide can be evaluated using the nonlinear parameter, defined as:

$$
\gamma(\omega)=\omega \frac{n_{2}}{c A_{e f f}}+i \frac{\beta_{T P A}}{2 A_{e f f}}
$$

where $n_{2}$ is the nonlinear refractive index, $A_{\text {eff }}$ the effective area and $\beta_{T P A}$ the TPA coefficient. The first term (also known as $R e \gamma$ ) of the equation represents the Kerr response of the waveguide and introduces a phase shift on the propagating light. The second term (also known as $I m \gamma$ ) is the TPA response, that quantifies the strength of the power-dependent nonlinear losses imposed to the optical signal.

$R e \gamma$ coefficients for the various waveguides were assessed by adopting a CW-FWM-based scheme [28]. The experimental set-up adopted for this measurement campaign is shown in Fig. 5 (a). Here a pump laser (set at a wavelength of $\lambda_{\mathrm{p}}$ $=1550.11 \mathrm{~nm}$, power ranging from $20 \mathrm{~mW}$ to $2 \mathrm{~W}$ before the waveguide input grating coupler) was amplified by a polarization maintaining Erbium-doped fibre amplifier (PMEDFA) and was sent to an optical band pass filter (BPF) to remove the amplified spontaneous emission (ASE), originated by the EDFA. The BPF showed a $3 \mathrm{~dB}$ bandwidth of $0.078 \mathrm{~nm}$ and a $10 \mathrm{~dB}$ bandwidth of $0.145 \mathrm{~nm}$. A fibre coupler (50:50) was used to combine the pump signal with a weaker $\mathrm{CW}$ light beam originated from a tuneable external cavity laser (ECL) and set at a wavelength of $\lambda_{\mathrm{s}}=1549.99 \mathrm{~nm}$. The optical power of the signal beam $\left(\lambda_{\mathrm{s}}\right)$ was always kept at least $10 \mathrm{~dB}$ below the power level of the pump $\left(\lambda_{\mathrm{p}}\right)$. The two waves were coupled to the waveguide under test by means of grating coupler devices. FWM in the waveguide generated an idler at a new frequency and all waves were coupled back to a PM fibre through the output grating coupler. Spectra were

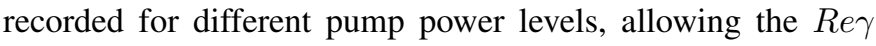



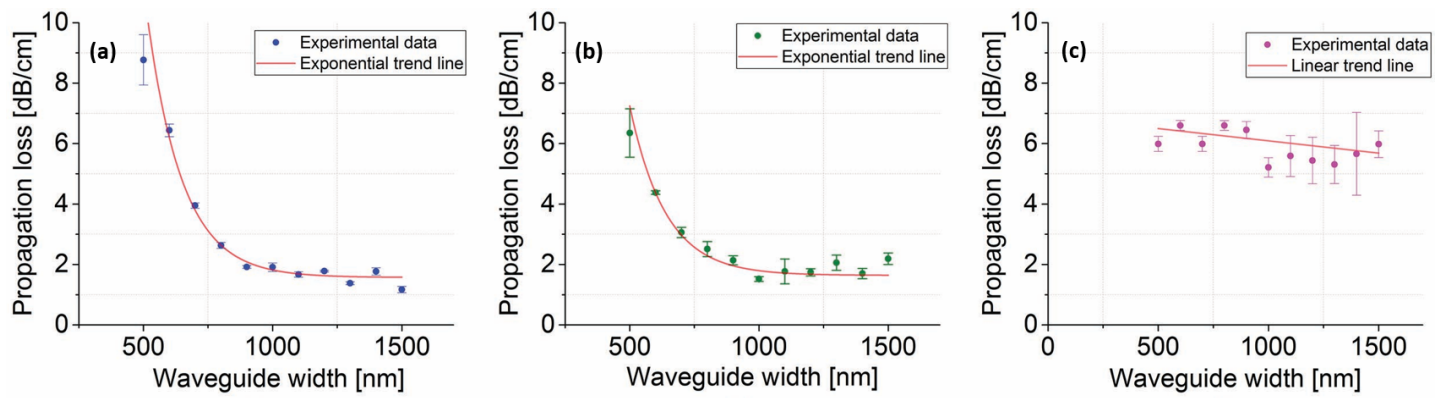

Fig. 4. Waveguide propagation losses measured for the three SiN layers: (a) propagation losses measured on waveguides written onto Layer ID 1, (b) layer ID 2 and (c)layer ID 3. Figure taken from [28]
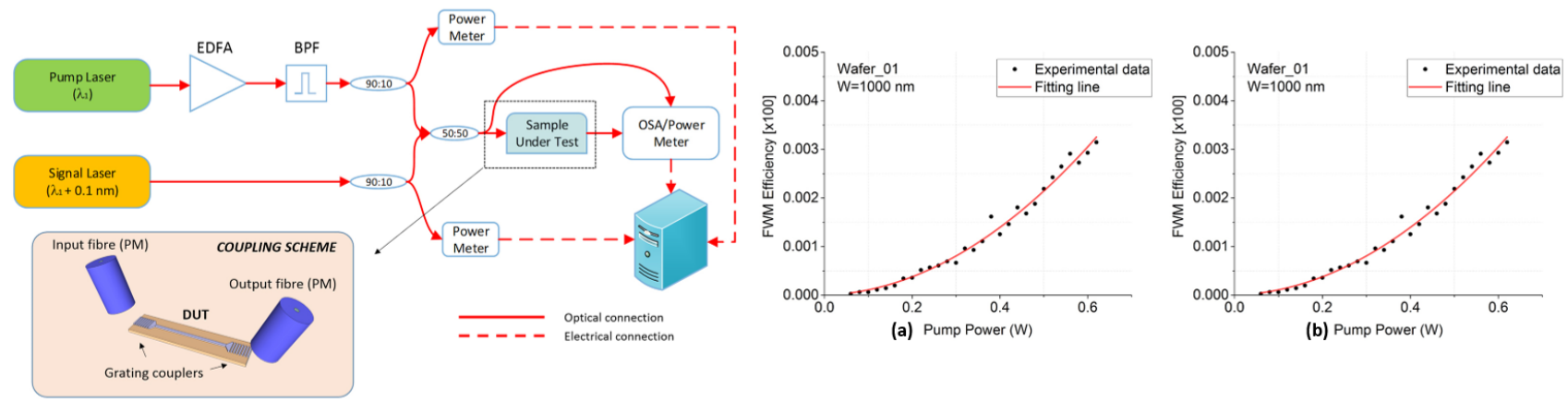

Fig. 5. (a) CW-FWM experimental set-up; BPF: Band Pass Filter; (b) example of FWM-efficiency curve versus optical power used to calculate the Rer coefficients (Layer ID 01, W=1000 nm); (c) example of CW-FWM spectrum recorded at the output of the waveguide (Layer ID 02, W=1000 nm, Pp=320 mW);

coefficients of the fabricated devices to be extracted, according to the following formula

$$
\operatorname{Re} \gamma=\frac{\sqrt{P_{i}(L) / P_{s}(L)}}{\eta P_{p}(0) L_{e f f}}
$$

where $P_{i}(L)$ and $P_{s}(L)$ are the idler and signal power levels, measured at the output of the waveguide under test, respectively; $P_{p}(0)$ represents the pump power measured at the input of the waveguide, $L_{e f f}$ is the nonlinear effective length and $\eta$ accounts for the phase-mismatch induced by chromatic dispersion. By placing the signal and pump beams relatively close to each other in wavelength $(\Delta \lambda<0.15 \mathrm{~nm})$ the effect of dispersion can be neglected, thus allowing to consider $\eta=1$.

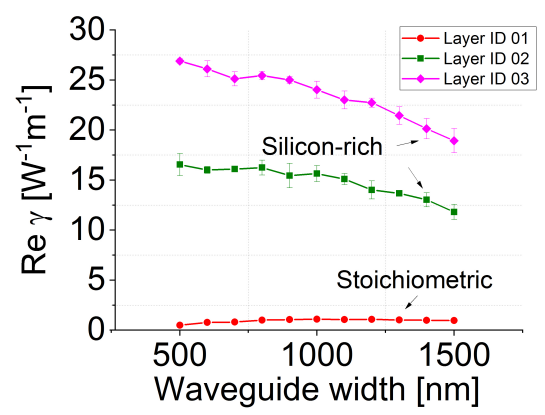

Fig. 6. Re $\gamma$ measured for each waveguide configuration, on each silicon nitride composition.

The results in Fig. 6 showed that Layer ID 02 and 03 exhibit enhanced nonlinear Kerr response (an order of magnitude higher than Layer ID 01). The observed enhancement is due to the increased material nonlinearities [28] induced by the presence of additional silicon within the material matrix. Thus, by properly tuning the silicon content in the $\mathrm{SiN}$ layers, the Rer coefficient can be made to approach values close to $30 \mathrm{Wm}^{-1}$ at a wavelength of $\lambda=1550 \mathrm{~nm}$. The imaginary part of $\gamma$ was assessed using pulse-transmission experiments [28]. To perform this experimental campaign a fibre mode locked laser centred at $1550 \mathrm{~nm}$ with pulse duration of $0.5 \mathrm{ps}$ and repetition rate of $20 \mathrm{MHz}$ was used as a light source. In order not to impose any spectral shaping on the input pulses, a butt-coupling scheme was employed in this case. The TPA-coefficients of the various waveguides were assessed by measuring the average power at the output of the sample under test as a function of the input peak power. By assuming a hyperbolic-secant pulse temporal profile, the average output power can be related to the input peak power through the following equation

$$
P(L)_{a v g}=\frac{\ln (\sqrt{\sigma}+\sqrt{\sigma+1})}{\sqrt{\sigma(\sigma+1)}} P(0)_{a v g} e^{-\alpha L}
$$

where

$$
\sigma=\frac{\beta_{T P A}}{A_{\text {eff }}} L_{e f f} P(0)_{\text {peak }}
$$

TPA-coefficients (thus $\operatorname{Im} \gamma$ ) were assessed using equations 3 and 4 . The obtained results for three different waveguide widths $(500,700$ and $1000 \mathrm{~nm})$ are summarised in Tab. III

Results revealed that both SiN layers ID 01 and ID 02 do not show significant TPA effects, even when relatively high power levels ( $>10 \mathrm{~W}$ peak power) are used. On the other hand, when the silicon content within the SiN matrix is significantly 
TABLE III

Im $\gamma$ COEFFICIENTS MEASURED FOR DIFFERENT SIN LAYER CONFIGURATIONS AND DIFFERENT WAVEGUIDE WIDTHS.

\begin{tabular}{llll}
\hline Layer ID & $\begin{array}{l}I m_{\gamma}\left[\mathbf{W m}^{-\mathbf{1}}\right] \\
\mathbf{W = 5 0 0} \mathbf{~ n m}\end{array}$ & $\begin{array}{l}I m_{\gamma}\left[\mathbf{W m}^{-\mathbf{1}}\right] \\
\mathbf{W = 7 0 0} \mathbf{n m}\end{array}$ & $\begin{array}{l}I m_{\gamma}\left[\mathbf{W m}^{-\mathbf{1}}\right] \\
\mathbf{W = 1 0 0 0 ~} \mathbf{n m}\end{array}$ \\
\hline 01 & Negligible & Negligible & Negligible \\
02 & Negligible & Negligible & Negligible \\
03 & 1.75 & 1.44 & 1.11 \\
\hline
\end{tabular}

increased (layer ID 03) TPA-related losses start to appear, thus indicating that this configuration is not suitable for high power low loss applications.

\section{III. (De)Multiplexing}

High-speed telecommunication requires wavelength division (de)multiplexing devices (WDM) with low insertion loss (IL), low cross-talk (XT), high tolerance to fabrication errors, and low sensitivity to temperature variations. SiN has surged as an alternative to fabricate WDM devices with improved performance and high tolerance to fabrication errors due to its smaller refractive index contrast compared to silicon [30][32]. It offers a good compromise between footprint and efficiency that enables achieving low IL and XT with relatively compact devices that are highly tolerant to temperature variations. In this section, we discuss the use of N-rich $\mathrm{SiN}$ films with a refractive index of 1.92 and thickness of $600 \mathrm{~nm}$ for the realisation of a 8-channel (de) multiplexer based on angled-multimode interferometers (AMMI) optimised for coarse WDM in the O-band (1260-1320 nm).

AMMIs have a lower channel count compared to arrayed waveguide gratings (AWG) and planar concave gratings (PCG). The interleaved AMMI (IAMMI) illustrated in Fig. 7 (a) distributes the resonant wavelengths of a Mach-Zendher interferometer (MZI) into two AMMIs to double their channel count [33]-[35]. Each of the AMMIs consists of a multimode dispersive waveguide of width $W_{M D W}$ with input/output waveguides of width $W_{I O}$ that are tilted at an angle $\theta_{t}$ and tapered from their single-mode width to $W_{a}$ [35], [36].

The dimensions of the two 4-channel AMMIs summarised in Table IV were optimised to obtain a spectral response with channel spacing $\sim 20 \mathrm{~nm}, \mathrm{IL}<1 \mathrm{~dB}$ and XT $<15 \mathrm{~dB}$ using FIMMWAVE following the procedure in [37]. The axial positions of the 8 outputs $\left(L_{i}\right)$ were calculated considering the interleaving condition given by equation 5 [33].

$$
\begin{gathered}
\lambda_{2 n}=\lambda_{2 n-1}+\frac{\Delta \lambda_{A M M I}}{2} \\
F S R=\frac{\lambda^{2}}{n_{g} \Delta L} \\
\lambda_{i}=\frac{2 n_{g} \Delta L}{m+1}
\end{gathered}
$$

For the MZI interleaver, $1 \times 2$ and $2 \times 2$ multi-mode interferometers (MMI) were optimised using Lumerical MODE Solutions to provide wavelength independent operation and IL $<0.1 \mathrm{~dB}$ in the O-band. Furthermore, the arm length difference of the MZI $(\Delta \lambda=46 \mu \mathrm{m})$ was calculated using equations 6 and 7, so that its free-spectral range (FSR) and peak resonant wavelengths matched the channel spacing and output wavelengths of the individual AMMIs.

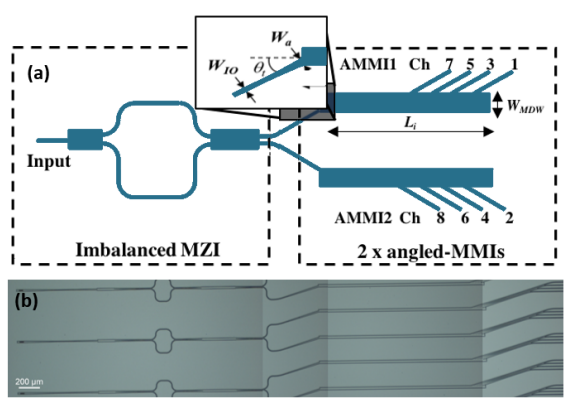

Fig. 7. (a) Schematic of an 8-channel interleaved AMMI and (b) microscopic images of the fabricated IAMMIs.

TABLE IV

OPTIMISED DESIGN PARAMETERS FOR THE 4-CHANNEL AMMIS.

\begin{tabular}{cccccc}
\hline \multicolumn{5}{c}{$W_{M D W}=18 \mu \mathrm{m}, W_{a}=7.5 \mu \mathrm{m}, \theta_{t}=0.3 \mathrm{rad}$} \\
\hline \multirow{2}{*}{ AMMI1 } & $\lambda_{\mathrm{i}}[\mathrm{nm}]$ & 1271 & 1291 & 1311 & 1331 \\
& $\mathrm{~L}_{\mathrm{i}}[\mu \mathrm{m}]$ & 1611 & 1584 & 1557 & 1530 \\
\hline \multirow{2}{*}{ AMMI2 } & $\lambda_{\mathrm{i}}[\mathrm{nm}]$ & 1281 & 1301 & 1321 & 1341 \\
& $\mathrm{~L}_{\mathrm{i}}[\mu \mathrm{m}]$ & 1598 & 1571 & 1543 & 1516 \\
\hline
\end{tabular}

The devices in figure 7 (b) were fabricated on $600 \mathrm{~nm}$ N-rich SiN films following the process outlined in Fig. 2. Fig. 8 (a) shows the spectral response of stand-alone AMMIs compared to the spectral response of the MZI. Both the $\Delta \lambda$ of the AMMIs and the FSR of the MZI have a mean value of $19.6 \mathrm{~nm}$, while their central wavelengths differ in $< \pm 1 \mathrm{~nm}$. Similarly, the spectral response of the AMMIs exhibits IL 1.0-1.5 dB, a $3 \mathrm{~dB}$ bandwidth of $(11.0 \pm 0.5) \mathrm{nm}$, and XT between $15-30 \mathrm{~dB}$ across the 4 channels. This response matches closely the required specification and is also highly tolerant to fabrication and temperature variations with spectral shifts of $114 \mathrm{pm} / \mathrm{nm}$ and $18 \mathrm{pm} /{ }^{\circ} \mathrm{C}$, respectively. The spectral response of the IAMMI in Fig. 8 (b) exhibits a $\Delta \lambda \sim(9.8 \pm 0.3) \mathrm{nm}$ with a deviation of $<1 \mathrm{~nm}$ from the target wavelengths. The $(8.0 \pm 0.7) \mathrm{nm} 3 \mathrm{~dB}$ bandwidth of the IAMMI is smaller than that of the stand-alone AMMIs due to the decreased bandwidth of the MZI, while its XT and IL increase to $\sim 12-20 \mathrm{~dB}$ and $\sim 3.5 \mathrm{~dB}$. The major contribution to the IL are the losses of the MZI, whereas the non-uniformity $\sim 0.5 \mathrm{~dB}$ across the channels can be attributed to the peak mismatch between the individual AMMIs and the MZI.

The peak wavelengths of all the devices drift towards longer wavelengths when the temperature is increased between 20$60^{\circ} \mathrm{C}$ as illustrated in Fig. 8 (c). The MZI suffers a shift of $15 \mathrm{pm} /{ }^{\circ} \mathrm{C}$, which is $3 \mathrm{pm} /{ }^{\circ} \mathrm{C}$ lower than that suffered by the AMMIs. We believe this is due to the greater mode overlap between the MZI single-mode waveguides and the cladding, compared to the multi-mode waveguides of the AMMIs. The temperature sensitivity of the final device $\sim 20 \mathrm{pm} /{ }^{\circ} \mathrm{C}$ is only $10 \%$ higher than that of the individual AMMIs. Thus, the 

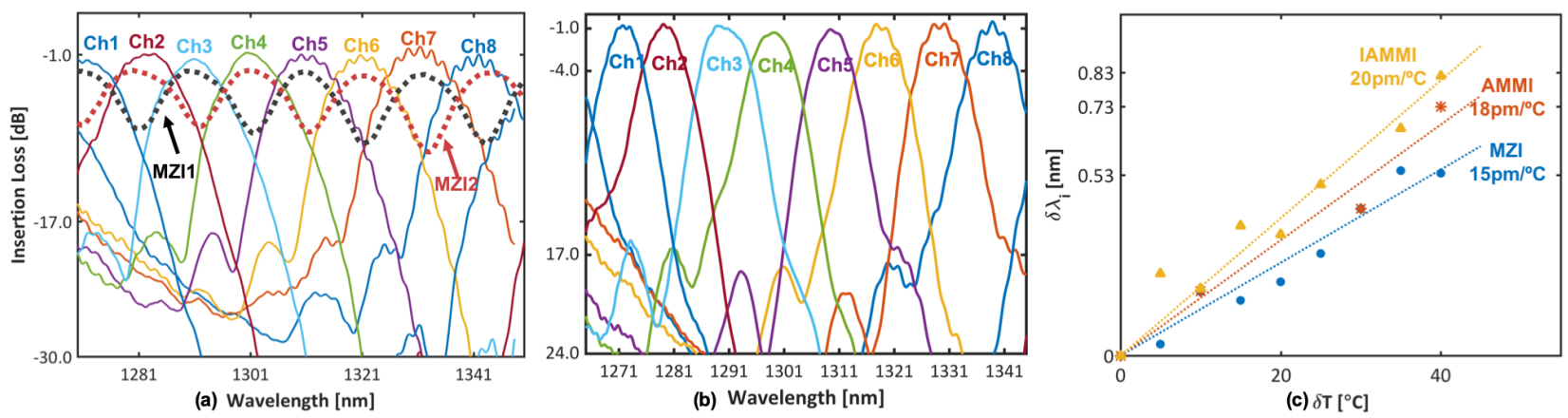

Fig. 8. Spectral responses of: (a) Stand-alone 4-channel AMMIs and MZI, and (b) the integrated 8-channel IAMMI normalised to the response of the MZI. (c) Wavelength shift $\left(\Delta \lambda_{i}\right)$ observed on the individual MZI, AMMIs and the complete IAMMI with different temperature variations $(\Delta T)$

IAMMI is still a robust option for applications that require a high tolerance to temperature variations.

Table V shows that the performance of both N-rich SiN AMMI and IAMMI is comparable with that of other SOI and SiN CWDM devices with the advantage of having a simple fabrication process, high tolerance to temperature variations and compatibility with multilayer integration due to the low processing temperature offered by the N-rich SiN deposition process. The most outstanding limitation of the IAMMI is the measured insertion loss, which is higher than typical values observed in other SiN CWDM devices due to the introduction of the MZI element. However, there is room to improve the performance of the device by optimising further the MMI and MZI elements to achieve lower loss contributions.

TABLE V

CWDM DEVICES DEMONSTRATED ON (A) SOI AND(B) SIN.

\begin{tabular}{cccccc}
\hline Device & $\begin{array}{c}\text { Footprint } \\
{\left[\mathrm{mm}^{2}\right]}\end{array}$ & $\begin{array}{c}\text { Ch. } \Delta \lambda \\
{[\mathrm{nm}]}\end{array}$ & $\begin{array}{c}\mathrm{IL} \\
{[\mathrm{dB}]}\end{array}$ & $\begin{array}{c}\mathrm{XT} \\
{[\mathrm{dB}]}\end{array}$ & $\begin{array}{c}\delta \lambda / \delta \mathrm{T} \\
{\left[\mathrm{pm} /{ }^{\circ} \mathrm{C}\right]}\end{array}$ \\
\hline (a) SOI \\
\hline AWG [38] & $0.55 \times 0.85$ & $4 \times 20$ & $<5.0$ & $\sim 17$ & \\
PCG [39] & $0.70 \times 0.39$ & $8 \times 6.2$ & $<1.0$ & 19 & $70-80$ \\
AMMI [36] & $0.02 \times 1.20$ & $4 \times 21.0$ & 2.0 & 20 & \\
IAMMI [33] & $0.05 \times 5.00$ & $8 \times 4.5$ & $<4.0$ & $15-20$ & \\
\hline \multicolumn{7}{c}{ (b) SiN } & & \\
\hline AWG [30] & $0.70 \times 3.70$ & $8 \times 20$ & 2.1 & 15 & $11-20$ \\
PCG [31] & - & $10 \times 6$ & 1.5 & 34 & $11-20$ \\
AMMI [37] & $0.02 \times 1.70$ & $4 \times 20$ & $<1.5$ & $16-30$ & 18 \\
IAMMI & $0.06 \times 3.00$ & $8 \times 9.8$ & $<4.0$ & $12-20$ & 20 \\
\hline
\end{tabular}

\section{All Optical Wavelength Conversion}

Modern optical communications require advanced functionalities to be applied on complex signals at ultrafast speeds. All-optical signal processing techniques offer a route to the implementation of such functionalities thus alleviating the need to convert the signals into the electrical domain, and offering potential cost savings. The developed SiN material, optimized for nonlinear applications, has been employed to design alloptical wavelength converters, able to process intensity and phase-encoded telecommunication signals. In this section, we discuss the application of dispersion-engineered silicon-rich silicon nitride waveguides to the realization of wavelength conversion of a 16 Quadrature Amplitude Modulation (QAM) signal operating at $10 \mathrm{Gbaud}(40 \mathrm{~Gb} / \mathrm{s})$. Bit Error Ratio (BER) measurements were carried out on the converted signal and revealed a power penalty of less than $1 \mathrm{~dB}$ with respect to the Back to Back (B2B) configuration. Continuous Wave (CW) measurements were also carried out on the device, indicating that the conversion bandwidth exceeded $40 \mathrm{~nm}$ and that no TPA-related effects were present, even at relatively high pump power operation $(27 \mathrm{dBm})$. The waveguide configuration utilized in this work is shown in Fig. 9 (a).

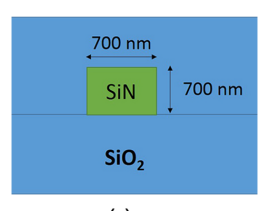

(a)

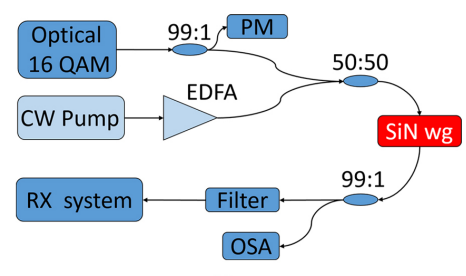

(b)
Fig. 9. (a) Waveguide cross section (b) wavelength converter experimental set-up.

The device was designed to operate as a broadband converter in the C-band. Numerical simulations were carried out to design waveguides with low dispersion and dispersion slope in this wavelength region. We opted for a waveguide width of $700 \mathrm{~nm}$, which showed a zero dispersion wavelength of $1550.20 \mathrm{~nm}$ with a variation of the $D$ parameter of less than $200 \mathrm{ps} / \mathrm{nm} / \mathrm{km}$ across the whole C-band. The waveguide was fabricated following the protocols detailed in previous sections and had a length of $10 \mathrm{~mm}$. The device included linear tapers to allow for coupling between the sample under test and tapered optical fibres. The coupling loss was $6 \mathrm{~dB} /$ facet, while the propagation loss measured using the Fabry-Perot fringes method, was $1 \mathrm{~dB} / \mathrm{cm}$. CW-FWM experiments were carried out in order to assess the conversion efficiency as a function of the injected pump power and conversion bandwidth. The results are shown in Fig. 10 (a) and Fig. 10 (b), respectively. The nonlinear parameter Re $\gamma$ was assessed from the FWM conversion efficiency measurements to be $12.5 \mathrm{Wm}^{-1}$ while the conversion bandwidth was flat across a $40 \mathrm{~nm}$ wavelength range around a wavelength of $1550 \mathrm{~nm}$. No TPA saturation was observed (see Fig. 10(a)), even when the pump power levels inside the waveguides exceeded $27 \mathrm{dBm}$. 
The experimental set-up used to perform the wavelength conversion experiments is shown in Fig. 9 (b). A 16 QAM signal $(40 \mathrm{~Gb} / \mathrm{s})$ was generated and combined with a $\mathrm{CW}$ pump by means of a 50:50 coupler. All components and fibers were polarization-maintaining, and both of the signal and the pump were linearly polarized (aligned to the slow axis of the fiber). The combined optical beam was then coupled to the silicon-rich silicon nitride waveguide, taking care to ensure that the slow fiber axis was aligned to the horizontal axis of the waveguide, thus only exciting the TE waveguide mode. The pump power coupled into the waveguide was $26 \mathrm{dBm}$ $(410 \mathrm{~mW})$. At the output of the waveguide, the pump and the signal were filtered out using a tunable, $1-\mathrm{nm}$ bandpass filter, while the converted signal was sent to the receiver. The RX system comprised a variable optical attenuator, a noise-loading unit and an Optical Modulation Analyzer (OMA), which allowed us to record BER measurements and constellation diagrams. Fig. 11 (a) shows an optical spectrum retrieved after the waveguide propagation, revealing a conversion efficiency of $-24 \mathrm{~dB}$. In Fig. 11 (b) we report on the BER results for the B2B configuration (blue circles) and converted signals detuned by $1 \mathrm{~nm}$ (red triangles) and $15 \mathrm{~nm}$ (green triangles) from the pump beam, respectively. In both cases, the measured BER curves of the converted signals showed a power penalty of less than $1 \mathrm{~dB}$ with respect to the B2B configuration. Fig. 11 (c) shows constellation diagrams of the original and the converted signals at a $\mathrm{BER}=10^{-6}$ confirming the negligible signal degradation.
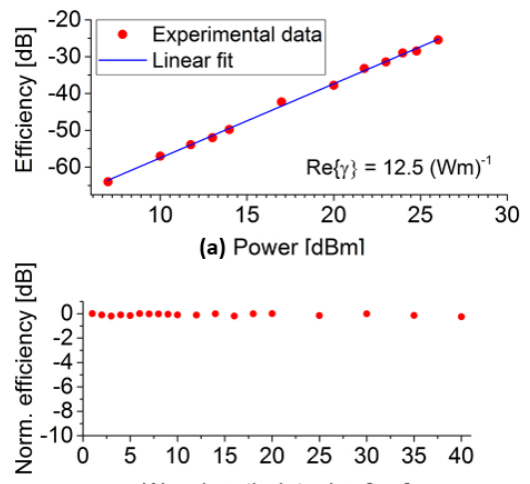

(b) Wavelength detuning [nm]

Fig. 10. (a) FWM efficiency as a function of the pump power; (b) FWM conversion bandwidth.

\section{Photonic CRystals}

Two-dimensional photonic crystal $(\mathrm{PhC})$ structures provide an effective way to tailor the propagation of light in a medium by introducing a periodic modulation of the refractive index [40]. In analogy with the case of crystalline solids, a periodic dielectric lattice enables a description of the light dispersion within the structured medium featuring photonic bands and, remarkably, the emergence of a spectral region where light propagation is inhibited, known as photonic bandgap (PBG) [41]. By engineering the design of these peculiar metamaterials, it is possible to achieve extremely high values of field enhancement with a consequent improvement of the
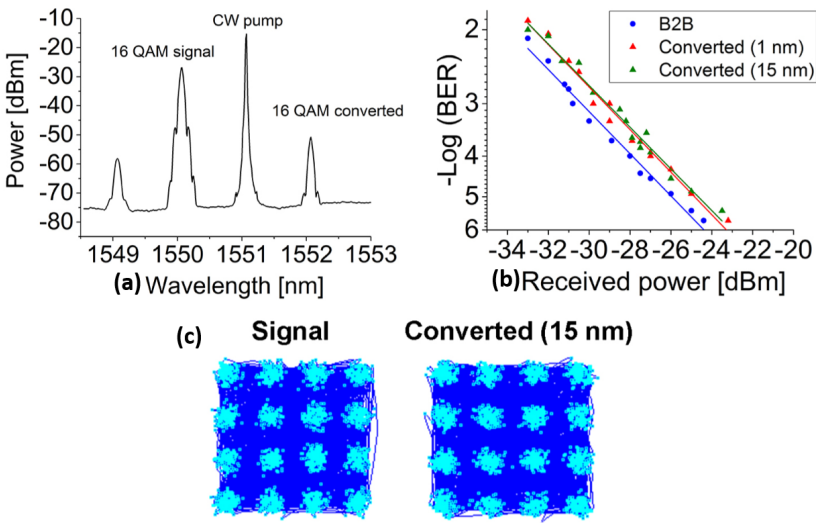

Fig. 11. (a) Example spectrum at the output of the device; (b) measured BER curves; (c) constellation diagrams of the original and converted signals.

light-matter interaction. This feature, achieved in resonant and non-resonant $\mathrm{PhC}$-based structures, provides benefits in a wide range of applications, such as nanolasers [42], frequency conversion [43], [44], all-optical switching [45], cavity QED [46], and nonlinear photonics in general. Specifically, lightmatter interaction can be enhanced in non-resonant structures via structural slow-light in $\mathrm{PhC}$ waveguides [47], while optical microcavities based on point defects in 2-dimensional PhCs provide a way to resonantly enhance the nonlinear interaction by confining the field to diffraction limited mode volumes (V) with extremely high quality factors (Q).

\section{A. Photonic Crystal Waveguides}

With the aim of exploiting the large nonlinear response of the Si-rich silicon nitride, we employed this material for the fabrication of $\mathrm{PhC}$ waveguides [48] and high-Q PhC microcavities [49], [50]. The $\mathrm{PhC}$ membrane was obtained in a $300 \mathrm{~nm}$ thick film of material with a refractive index $n=2.48$ patterned with a triangular lattice of air holes of period $a=580 \mathrm{~nm}$ and radius $r=0.3 a$. This choice of parameters provided a $210 \mathrm{~nm}$ wide $\mathrm{PBG}$ centered at $1475 \mathrm{~nm}$ and extending over the full III window of telecom wavelengths, as predicted by plane wave expansion (PWE) calculations and later confirmed experimentally. The deposited film was patterned by means of electron beam lithography combined with a inductively coupled plasma reactive ion etching (ICPRIE) process and then suspended by wet etching of the silicon substrate in a Tetra-methyl-ammonium hydroxide (TMAH) aqueous solution.

The $\mathrm{PhC}$ waveguides were implemented by introducing a line defect, consisting of a removed line of holes in the $\Gamma$ $\mathrm{K}$ direction (W1 waveguide). In this structure we demonstrated a maximum group index of over 110, although the best value of $n_{g}$ was associated to extremely high scattering losses $(>100 \mathrm{~dB} / \mathrm{cm})$. A group index $n_{g}=37$ was reported in combination with a more acceptable value of propagation loss of $53 \mathrm{~dB} / \mathrm{cm}$. Given the small length of the devices, this value points to a good performance for applications such as integrated delay lines and nonlinear photonic devices based on slow-light. By tailoring the width of the $\mathrm{PhC}$ waveguide, we were able to achieve a region of low propagation loss 
$(4.6 \mathrm{~dB} / \mathrm{cm})$ and group index $n_{g}=7.4$ over a bandwidth of over $70 \mathrm{~nm}$. Since the intensity enhancement provided by slowlight is expected to scale proportionally with the group index $n_{g}$ [51], PhC waveguides provide a way to exploit effectively the improved nonlinear properties of the TPA-free, silicon-rich nitride material in a compact device footprint.

\section{B. Photonic Crystal Cavities}

$\mathrm{PhC}$ cavities provide one of the most effective ways to enhance light-matter interaction, with immediate advantages for nonlinear applications. The intensity enhancement effect is expected to scale with the ratio $Q / V$, which in this kind of structures is among the highest reported in the literature enabling to exploit nonlinear optical processes even at extremely low input power and continuous wave $(\mathrm{CW})$ regime [52]. The applications of $\mathrm{PhC}$ cavities to nonlinear optics have been widely studied in the field of silicon photonics [53], where TPA is however detrimental for practical applications. Here we report the implementation of $\mathrm{PhC}$ cavities in Si-rich silicon nitride and briefly review their application for lowpower, on-chip frequency conversion via second- and thirdharmonic generation.

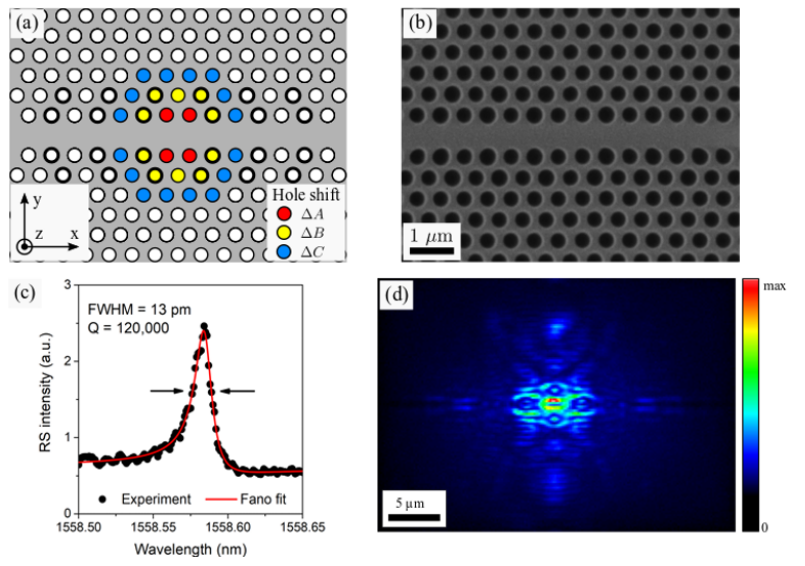

Fig. 12. (a) PhC cavity design. The positions of the holes marked in red, yellow and blue are shifted aside the waveguide in the $y$-direction to achieve gentle confinement. The holes marked in bold are slightly enlarged in the cavities designed for harmonic generation, in order to optimize the coupling efficiency to free space. (b) SEM picture of one of the fabricated devices. (c) RS spectrum of a $\mathrm{PhC}$ microcavity optimized for ultrahigh $\mathrm{Q}$ factor. (d) Near-field image (false colors) of the intracavity generated second harmonic.

We designed our PhC cavities [49] starting from a W1 waveguide patterned with the geometry described in the previous section, where specific holes where shifted away from the waveguide in the transverse direction (colormarked holes in fig. 12 (a)) by $\Delta A=15 \mathrm{~nm}, \Delta B=10 \mathrm{~nm}$ and $\Delta C=5 \mathrm{~nm}$. This design, known as Al cavity [54], leads to a $\mathrm{Q}$ factor as high as 520.000 and a mode volume $V=\int \varepsilon|E|^{2} d^{3} r / \max \left\{\varepsilon|E|^{2}\right\}=0.77(\lambda / n)^{3}$, as estimated from finite-difference time-domain (FDTD) simulations, almost diffraction limited and yet unparalleled by traveling-wave resonators such as microrings or microtoroids. We characterized the fabricated devices (fig. 12 (b)) by means of resonant scattering (RS) technique [55], through which we measured intrinsic Q factors ranging from 71.000 to 122.000 (fig. 12 (c)). The discrepancy with the numerical estimate is mainly due to the residual linear absorption of the material, which limits the maximum ring-down time of the cavity mode.

In order to probe the nonlinear optical properties of these structures, we produced samples optimized for efficient second- and third-harmonic generation (HG) [50]. By appropriately adjusting the radius of specific holes (marked bold in Dig. 12 (a)) [56], we were able to tailor the coupling efficiency $\eta_{c}=P_{\text {coupled }} / P_{\text {incident }}$ of a Gaussian transverse optical mode orthogonally impinging from free space on the device. Here, the efficiency of the $n$-th order HG process is predicted to scale as the figure of merit $\left(\eta_{c} Q / V\right)^{n}$. For this reason, we chose a value of $\Delta r=+12 \mathrm{~nm}$ for our farfield optimization, leading to a coupling efficiency as high as $\eta_{c}=30 \%$. With this strategy, we reduced the $\mathrm{Q}$ factor of the cavity to 12.000 but we maximized at the same time the figure of merit for frequency conversion. The wide bandgap of the material provides transparency at the second harmonic $(\mathrm{SH})$ wavelength (for our experiments, $\lambda_{S H}=771 \mathrm{~nm}$, with pump in the telecom band resonant with the fundamental cavity mode). A near-field image of the generated $\mathrm{SH}$ is depicted in Fig.12 (d). Despite the amorphous nature of the bulk material yields a negligible $\chi^{(2)}$ nonlinear response, the $\mathrm{SH}$ generation process is here still appreciable thanks to the breaking of inversion symmetry provided by the presence of multiple interfaces air/dielectric [52]. The simultaneous generation of third harmonic (TH) is also observed, although the strong linear absorption of the material at $\lambda_{T H}$ practically limits the applications of the process. From calibrated measurements of the generated $\mathrm{SH}$ and $\mathrm{TH}$ power, we estimated the efficiency of the $\mathrm{SH}$ and $\mathrm{TH}$ generation processes to be respectively $\rho_{S H}=P_{S H} / P_{\text {coupled }}^{2}=(4.7 \pm 0.2) \times 10^{-7} \mathrm{~W}^{-1}$ and $\rho_{T H}=P_{T H} / P_{\text {coupled }}^{3}=(5.9 \pm 0.3) \times 10^{-5} \mathrm{~W}^{-2}$. Remarkably, our results highlight the absence of TPA, overcoming the limitations of narrow bandgap materials for nonlinear applications in a CMOS-compatible platform.

\section{Novel Material Integration}

Although silicon nitride is a flexible material [57], [58], it exhibits different challenges that need to be addressed such as its lack modulation mechanism. This situation has driven interest in hybrid silicon photonic devices in which optical functionality is provided by a secondary material, such as 2D materials [59], liquid crystals [60], metals [61], electrooptic polymers [62] [63], germanium [64], and optical phase change materials [PCMs] [65]-[67]. This section presents the large scale integration of novel materials on $\mathrm{SiN}$ devices including experimental work integrating O-PCMs for a variety of functionalities.

Optical phase-change materials (PCMs) have emerged as a unique class of materials that have two phases (amorphous and crystalline) that exhibit large changes in their optical properties ( $\Delta n>1, \Delta \kappa \sim$ order of magnitude). These two phases are reversible making PCM promising candidates to address non volatile re-configurability [68], [69]. The phase change can be induced thermally, optically or electrically and in all cases high-speed switching can be achieved ( ns) [70], [71]. The 
most widely studied PCMs are transition metal oxides and chalcogenid-based alloys. These materials have been exploited for a variety of tunable photonic applications ranging from smart windows [72] to metamaterials [73]. The combination of $\mathrm{SiN}$ waveguides and PCMs can lead to ultrafast non volatile re-configurable technology for optical communications applications [74], [75].
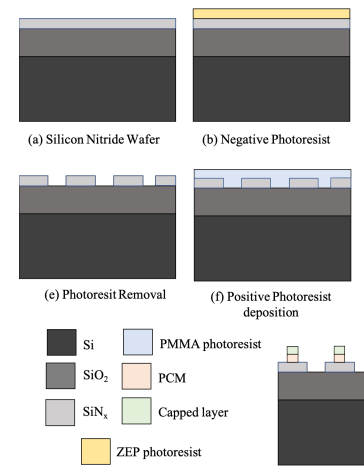

esist
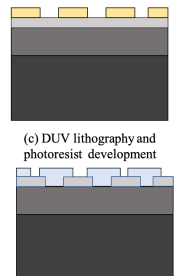

(g) DUV lithography and (g) DUV lithography and
photoressist developmen

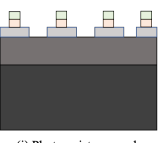

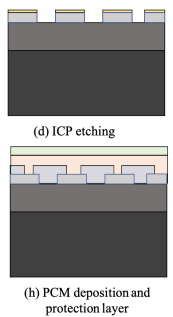

Fig. 13. Large scale fabrication process (a) Silicon Nitride wafer (b) spin coating negative photoresist (c) DUV lithography (d) ICP etching (e) Photoresist removal (f) Positive photoresist deposition (g) DUV lithography and photoresist removal (h) PCM deposition

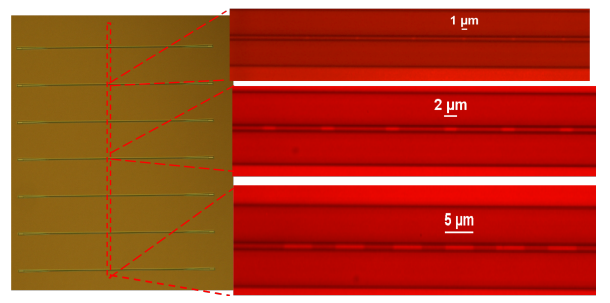

Fig. 14. Optical microscope images of the straight waveguides with a 1,2 and 5 um PCM of GST patch deposited on top of the structure. Zoom in red of a microscope image verifying the length of the phase change material patch for the different configurations.

A large scale fabrication process for re-configurable integrated devices based on phase change materials has been demonstrated (Fig.13). A variety of integrated devices, including waveguides and MZI, were fabricated on a $600 \mathrm{~nm}$ thick $\mathrm{N}$-rich $\mathrm{SiN}_{\mathrm{x}}$ layer with a refractive index of $1.92(\lambda=1310 \mathrm{~nm})$ deposited on 8 inch silicon wafers $(200 \mathrm{~mm}$ with a $2 \mu \mathrm{m}$ thermally grown $\mathrm{SiO}_{2}$ layer. The device layout was defined on the wafer using deep ultraviolet lithography and transferred to the SiN layer with ICP etching. Once the devices were fabricated, the wafer was diced into samples to create windows using electron beam lithography with a MMA+PMMA photoresist for the deposition of the phase change material. These windows had different lengths varying from 2 to $10 \mu \mathrm{m}$ in order to change the length of the PCM patch deposited on top of the structures. After developing the resist, the GST phase change material was sputtered on top of the samples and the MMA+PMMA resist was removed to create the PCM patches as shown in Fig. 14.

By changing the state of the PCM from amorphous to crystalline, it is possible to induce an effective index change that enables the realization of switches. In this work, an optical switch based on a MZI has been demonstrated for the O-band. The arms of the MZI consisted of $700 \times 600 \mathrm{~nm}$ strip waveguides with a length difference of $40 \mu \mathrm{m}$. A $5 \mu \mathrm{m}$ GST patch was deposited on the longer arm of the MZI and the response of the device was characterized using a laser source with a wavelength tuning range between 1260 and $1320 \mathrm{~nm}$. The state of the PCM patch was then changed during the deposition of a PECVD oxide cladding at a temperature of $350{ }^{\circ} \mathrm{C}$. Fig. 15 shows the response of the MZI for the amorphous and crystalline phase of the PCM patch. It can be observed that the device acts as a switch in the O-band with an extinction ratio of $10.7 \mathrm{~dB}$ at $1295 \mathrm{~nm}$ and overall insertion loss of $5.5 \mathrm{~dB}$.

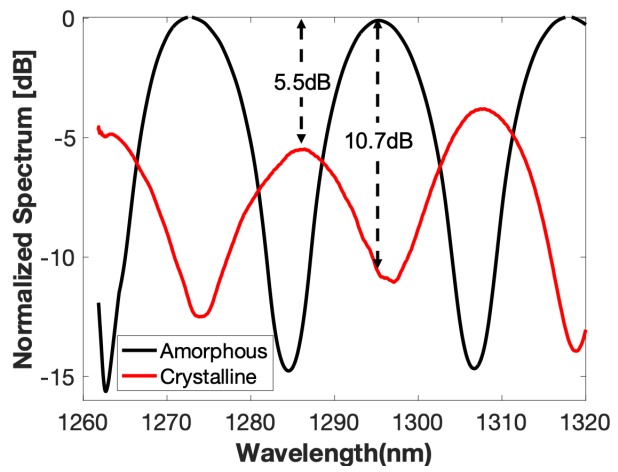

Fig. 15. Transmission for a MZI with $\Delta L=40 \mu \mathrm{m}$ and $L_{G S T}=5 \mu \mathrm{m}$ in the amorphous state (red line) and in the crystalline state (black line). The spectra are normalized respect to the transmission of the amorphous state.

\section{The $2 \mu \mathrm{m}$ WAVELEngth Regime}

As the data traffic increases, current telecommunications are approaching the capacity limit of conventional single mode fibres that operate in the $\mathrm{O}$ and $\mathrm{C}$ wavelengths bands. As a result, there is an increasing interest in wavelength ranges in which new technologies can be implemented to help overcome this limitation. The $2 \mu \mathrm{m}$ wavelength range has received particular attention as it benefits from the emergence of thulium-doped fibre amplifiers and hollow-core photonic bandgap fibres whose gain window and low loss $(0.1 \mathrm{~dB} / \mathrm{km})$ is centred around 1900-2100 $\mathrm{nm}$ [76]-[78]. In this context, silicon nitride is a material that has potential for applications in this new wavelength regime as it is transparent well within the limits of the $2 \mu \mathrm{m}$ wavelength band. In addition, it has the potential to exhibit a low thermo-optic coefficient and low propagation losses which are essential to demonstrate efficient devices with high tolerance to temperature variations.

To probe the performance and extend the use of our low temperature SiN platform to this emerging wavelength regime, we have designed single-mode waveguides optimised to operate at $2 \mu \mathrm{m}$. As illustrated in Fig. 16, the thickness of the waveguide core is bigger compared to the thicknesses typically used for the $\mathrm{O}$ and $\mathrm{C}(300-700 \mathrm{~nm})$ wavelength bands in order to accommodate the mode of the longer wavelength. The waveguides were fabricated on a $1000 \mathrm{~nm}$ N-rich SiN layer grown on 8 " wafers with a $3 \mu \mathrm{m}$ thermal $\mathrm{SiO}_{2}$ using the process described in Fig. 2. Again, the buried oxide is $1 \mu \mathrm{m}$ 
thicker than the one used at shorter wavelengths to ensure that there is no power leakage towards the substrate.

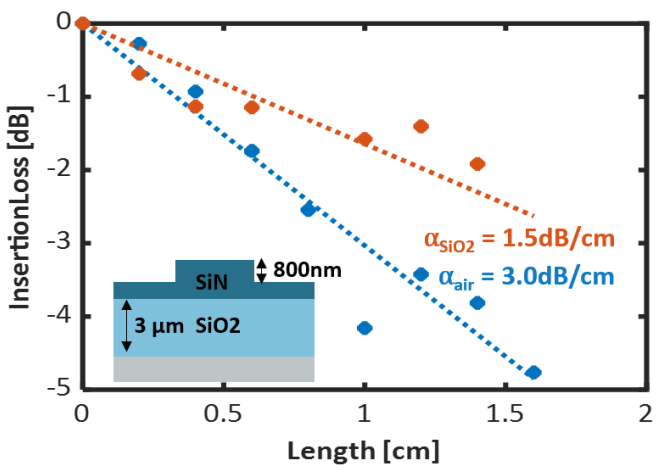

Fig. 16. Insertion loss as a function of waveguide length for air and $\mathrm{SiO}_{2}$ claddings.

As shown in Fig. 16, the propagation loss of the designed waveguide was determined using the cut-back method. The loss was estimated to be $3.0 \mathrm{~dB} / \mathrm{cm}$ with an air cladding. This value decreased to $\sim 1.5 \mathrm{~dB} / \mathrm{cm}$ when the waveguides were covered with a $\mathrm{SiO}_{2}$ cladding. We believe that the increased roughness of the thicker waveguide is a big contribution to the measured loss with an air cladding. A more detail study needs to be conducted to rule out other sources of losses and to optimise the material structure for this new wavelength. However, we have proved that there is potential to use the SiN platform for the fabrication of integrated devices for telecommunication and even sensing applications in this wavelength regime.

\section{CONCLUSION}

In this work, we have shown the flexibility of our low temperature silicon nitride platform and how its linear and nonlinear properties can be tailored for a wide variety of applications. We have demonstrated that with N-rich SiN it is possible to realise efficient CWDM devices with a low sensitivity to fabrication errors and high tolerance to temperature variations $\left(\sim 20 \mathrm{pm} /{ }^{\circ} \mathrm{C}\right)$. In a similar manner, we have shown that dispersion-engineered $\mathrm{Si}$-rich $\mathrm{SiN}$ waveguides have enhanced nonlinear properties that can be used for all optical wavelength conversion of modulated signals with negligible signal degradation (BER $\sim 10^{-6}$ ). Additionally, we have demonstrated photonic crystal waveguides that provide and efficient way to exploit the improved non-liner properties of Si-rich SiN, along with second- and third harmonic generation in photonic crystal cavities with no saturation effects related to two-photon absorption. We have introduced the integration of $\mathrm{SiN}$ with phase change materials to demonstrate more advance functionlaties such as switching that can be further extended to realise modulators. Finally, we have examined the propagation losses of N-rich SiN for applications at $2 \mu \mathrm{m}$.

Hopefully, the topics discussed in this work will serve to boost the usage of SiN for many other photonic applications and to push forward its use in complex photonic integrated circuits where it can be combined with other materials to tackle some of the challenges that CMOS based integrated photonics will need to address in the 21 st century.

\section{REFERENCES}

[1] B. Troia, A. Z. Khokhar et al., "Design procedure and fabrication of reproducible silicon vernier devices for high-performance refractive index sensing," Sensors, vol. 15, no. 6, pp. 13 548-13 567, 2015.

[2] A. K. Goyal, H. S. Dutta, and S. Pal, "Recent advances and progress in photonic crystal-based gas sensors," J. Phys. D. Appl. Phys., vol. 50, no. 20, p. 203001, 2017.

[3] S. Clemmen, P. Emplit, S. Massar, K. Phan-Huy, W. Bogaerts, and R. G. Baets, "Integrated quantum optics using the silicon on insulator (SOI) plateform," in FiO 2012/LS XXVIII. OSA, 2012, p. FTu4D.1.

[4] S. Zhu, G. Q. Lo, and D. L. Kwong, "Low-loss amorphous silicon wire waveguide for integrated photonics: effect of fabrication process and the thermal stability." Opt. Express, vol. 18, no. 24, pp. 25 283-25 291, 2010.

[5] S. K. Selvaraja, E. Sleeckx et al., "Low-loss amorphous silicon-oninsulator technology for photonic integrated circuitry," Opt. Cотmun., vol. 282 , no. 9 , pp. 1767-1770, 2009.

[6] T. Lipka, L. Moldenhauer, J. Müller, and H. K. Trieu, "Photonic integrated circuit components based on amorphous silicon-on-insulator technology," Photonics Res., vol. 4, no. 3, pp. 126-134, 2016.

[7] L. A. Eldada, "Advances in telecom and datacom optical components," Opt. Eng., vol. 40, pp. 14-40, 2001.

[8] G. L. Bona, R. Germann, and B. J. Offrein, "SiON high-refractive-index waveguide and planar lightwave circuits," IBM J. Res. Dev., vol. 47, no. 2.3, pp. 239-249, 2003.

[9] K. Worhoff, E. Klein, G. Hussein, and A. Driessen, "Silicon oxynitride based photonics," in 2008 10th Anniv. Int. Conf. Transparent Opt. Networks, vol. 3, 2008, pp. 266-269.

[10] S. Mao, S. Tao et al., "Low propagation loss SiN optical waveguide preopared by optimal low-hydrogen module," Opt. Express, vol. 16, no. 25, pp. 20809-20816, 2008.

[11] K. Wörhoff, R. G. Heideman, A. Leinse, and M. Hoekman, "TriPleX: a versatile dielectric photonic platform," Adv. Opt. Technol., vol. 4, no. 2, pp. 189-207, 2015.

[12] D. J. Blumenthal, R. Heideman, D. Geuzebroek, A. Leinse, and C. Roeloffzen, "Silicon nitride in silicon photonics," Proc. IEEE, vol. 106, no. 12, pp. 2209-2231, Dec 2018.

[13] R. Soref, "Mid-infrared photonics in silicon and germanium," Nat. Photonics, vol. 4, pp. 495-497pp, 2010.

[14] S. Romero-García, F. Merget, F. Zhong, H. Finkelstein, and J. Witzens, "Silicon nitride CMOS-compatible platform for integrated photonics applications at visible wavelengths." Opt. Express, vol. 21, no. 12, pp. 14036-46, 2013.

[15] W. H. P. Pernice, M. Li, D. F. G. Gallagher, and H. X. Tang, "Silicon nitride membrane photonics," J. Opt. A Pure Appl. Opt., vol. 11, no. 11, p. 114017, 2009.

[16] V. Verlaan, A. D. Verkerk et al., "The effect of composition on the bond structure and refractive index of silicon nitride deposited by HWCVD and PECVD," Thin Solid Films, vol. 517, pp. 3499-3502, 2009.

[17] A. Arbabi and L. L. Goddard, "Measurements of the refractive indices and thermo-optic coefficients of $\mathrm{Si} 3 \mathrm{~N} 4$ and $\mathrm{SiOx}$ using microring resonances," Opt. Lett., vol. 38, no. 19, pp. 3878-3881, Oct 2013.

[18] D. J. Moss, R. Morandotti, A. L. Gaeta, and M. Lipson, "New CMOScompatible platforms based on silicon nitride and Hydex for nonlinear optics," Nat. Photonics, vol. 7, no. 8, pp. 597-607, 2013.

[19] T. Domínguez Bucio, A. Z. Khokhar et al., "Material and optical properties of low-temperature NH3-free PECVD SiNx layers for photonic applications," J. Phys. D. Appl. Phys., vol. 50, no. 2, p. 025106, 2017.

[20] E. Bustarret, M. Bensouda, M. C. Habrard, J. C. Bruyére, S. Poulin, and S. C. Gujrathi, "Configurational statitistics in a-SixNyHz alloys: A quantitative bonding analysis," Phys. Rev. B, vol. 38, no. 12, pp. 81718184pp., 1988.

[21] Z. Yin and F. W. Smith, "Optical dielectric function and infrared absoprtion of hydrogenated amorphous silcon nitride films: Experimental results and effective-medium-approximation analysis," Phys. Rev. B, vol. 42 , no. 6, pp. 3666-3672, 1990

[22] H. Zhang, C. Li, X. Tu, X. Luo, M. Yu, and P. G. Lo, "High efficiency silicon nitride grating coupler," App. Phys. A, vol. 22, no. 18, pp. 21800 $21805,2013$.

[23] Y. Huang, J. Song, X. Luo, T.-Y. Liow, and G.-Q. Lo, "CMOS compatible monolithic multi-layer Si3N4-on-SOI platform for low-loss high performance silicon photonics dense integration," Opt. Express, vol. 22, no. 18, pp. $21859-21865,2014$.

[24] F. Karouta, K. Vora, J. Tian, and C. Jagadish, "Structural, composition and optical properties of PECVD silicon nitride layers," J. Phys. D: Appl. Phys., vol. 45, no. 44, p. 445301, 2012. 
[25] V. Verlaan, R. Bakker et al., "High density silicon nitride deposited at low substrate temperature with high deposition rate using hot wire chemical vapour deposition." Surf. Coat. Technol., vol. 201, no. 22-23, pp. 9285-9288, 2007

[26] A. Tarazona, T. D. Bucio et al., "Hot wire chemical vapor deposition for silicon photonics: An emerging industrial application opportunity," Thin Solid Films, vol. 676, pp. 26-30, 2019.

[27] N. Sherwood-Droz and M. Lipson, "Scalable 3D dense integration of photonics on bulk silicon," Opt. Express, vol. 19, no. 18, pp. 17758 17765, 2011.

[28] C. Lacava, S. Stankovic et al., "Si-rich Silicon Nitride for Nonlinear Signal Processing Applications," Sci. Rep., vol. 7, no. 1, 2017.

[29] C. Lacava, T. Dominguez Bucio et al., "Intermodal frequency generation in silicon-rich silicon nitride waveguides," Photonics Res., vol. 7, no. 6, p. $615,2019$.

[30] D. Dai, Z. Wang et al., "Low-loss Si3N4 arrayed-waveguide grating (de)multiplexer using nano-core optical waveguides," Opt. Express, vol. 19, no. 15, pp. 14 130-14 136, 2011.

[31] E. Ryckeboer, X. Nie et al., "CMOS-compatible silicon nitride spectrometers for lab-on-a-chip spectral sensing," Proc. SPIE, vol. 9891, pp. 98911K-98 911K-9, 2016.

[32] T. Domínguez Bucio, A. Z. Khokhar, G. Z. Mashanovich, and F. Y. Gardes, "Athermal silicon nitride angled MMI wavelength division (de)multiplexers for the near-infrared," Opt. Express, vol. 25, no. 22 pp. 27310-27320, oct 2017.

[33] Y. Hu, F. Y. Gardes, D. J. Thomson, G. Z. Mashanovich, and G. T Reed, "Coarse wavelength division (de)multiplexer using an interleaved angled multimode interferometer structure," Appl. Phys. Lett., vol. 102, no. 25 , pp. 7-11, 2013.

[34] Y. Hu, T. Li et al., "Mid-infrared wavelength division (de)multiplexer using an interleaved angled multimode interferometer on the silicon-oninsulator platform." Opt. Lett., vol. 39, no. 6, pp. 1406-9, 2014.

[35] Y. Hu, D. J. Thomson, F. Y. Gardes, G. Z. Mashanovich, , and G. T. Reed, "The evolution of angled mmi structure on the soi platform," in Proc. SPIE 8990, Silicon Photonics IX, vol. 8990, 2014.

[36] Y. Hu, R. Jenkins, and F. Y. Gardes, "Wavelength division (de) multiplexing based on dispersive self-imaging," Opt. Lett., vol. 36, no. 23, pp. 4488-4490, 2011.

[37] T. Domínguez Bucio, A. Z. Khokhar, G. Z. Mashanovich, and F. Y Gardes, "N-rich silicon nitride angled-MMI for coarse wavelength division (de)multiplexing in the O-band," Opt. Express, vol. 43, no. 5 , 2018.

[38] S. Dwivedi, P. De Heyn, P. Absil, J. Van Campenhout, and W. Bogaerts, "Coarse wavelength division multiplexer on silicon-on-insulator for 100 GbE," in IEEE Int. Conf. Gr. IV Photonics GFP, vol. r, 2015, pp. 9-10.

[39] S. Pathak, P. Dumon, D. Van Thourhout, and W. Bogaerts, "Comparison of AWGs and Echelle Gratings for Wavelength Division Multiplexing on Silicon-on-Insulator," IEEE Photonics J., vol. 6, no. 5, pp. 1-9, 2014.

[40] M. Notomi, "Manipulating light with strongly modulated photonic crystals," Rep. Prog. Phys., vol. 73, no. 9, p. 096501, sep 2010.

[41] J. D. Joannopoulos, S. G. Johnson, J. N. Winn, R. D. Meade, and C. J. Sjödahl, Photonic crystals: molding the flow of light. Princeton University Press, 2011.

[42] K. Nozaki, S. Kita, and T. Baba, "Room temperature continuous wave operation and controlled spontaneous emission in ultrasmall photonic crystal nanolaser," Opt. Express, vol. 15, no. 12, p. 7506, 2007.

[43] S. Azzini, D. Grassani et al., "Stimulated and spontaneous four-wave mixing in silicon-on-insulator coupled photonic wire nano-cavities," Appl. Phys. Lett., vol. 103, no. 3, 2013.

[44] M. S. Mohamed, A. Simbula et al., "Efficient continuous-wave nonlinear frequency conversion in high-Q gallium nitride photonic crystal cavities on silicon," APL Photonics, vol. 2, no. 3, p. 031301, 2017.

[45] K. Nozaki, T. Tanabe et al., "Sub-femtojoule all-optical switching using a photonic-crystal nanocavity," Nat. Photonics, vol. 4, no. 7, pp. 477483, jul 2010.

[46] K. Hennessy, A. Badolato et al., "Quantum nature of a strongly coupled single quantum dot-cavity system," Nature, vol. 445, no. 7130, pp. 896899, 2007.

[47] T. F. Krauss, "Slow light in photonic crystal waveguides," J. Phys. D. Appl. Phys., vol. 40, no. 9, pp. 2666-2670, 2007.

[48] K. Debnath, T. Dominguez Bucio, A. Al-Attili, A. Z. Khokhar, S. Saito, and F. Y. Gardes, "Photonic crystal waveguides on silicon rich nitride platform," Opt. Express, vol. 25, no. 4, p. 3214, 2017.

[49] K. Debnath, M. Clementi et al., "Ultrahigh-Q photonic crystal cavities in silicon rich nitride," Opt. Express, vol. 25, no. 22, p. 27334, 2017.
[50] M. Clementi, K. Debnath et al., "Cavity-enhanced harmonic generation in silicon rich nitride photonic crystal microresonators," Appl. Phys. Lett., vol. 114, no. 13, p. 131103, 2019

[51] R. W. Boyd, "Material slow light and structural slow light: similarities and differences for nonlinear optics," J. Opt. Soc. Am. B., vol. 29, no. 9, p. 2644, 2012.

[52] M. Galli, D. Gerace et al., "Low-power continuous-wave generation of visible harmonics in silicon photonic crystal nanocavities," Opt. Express, vol. 18 , no. 25 , p. $26613,2010$.

[53] J. Bravo-Abad, A. Rodriguez, P. Bermel, S. G. Johnson, J. D. Joannopoulos, and M. Soljacic, "Enhanced nonlinear optics in photonic-crystal microcavities," Opt. Express, vol. 15, no. 24, p. 16161, 2007.

[54] E. Kuramochi, M. Notomi, S. Mitsugi, A. Shinya, T. Tanabe, and T. Watanabe, "Ultrahigh-Q photonic crystal nanocavities realized by the local width modulation of a line defect," App. Phys. Lett., vol. 88, no. 4, p. 041112, 2006.

[55] M. Galli, S. L. Portalupi, M. Belotti, L. C. Andreani, L. O'Faolain, and T. F. Krauss, "Light scattering and Fano resonances in high-Q photonic crystal nanocavities," App. Phys. Lett., vol. 94, no. 7, pp. 1-3, 2009.

[56] S. L. Portalupi, M. Galli et al., "Planar photonic crystal cavities with far-field optimization for high coupling efficiency and quality factor," Opt. Express, vol. 18, no. 15, p. 16064, 2010.

[57] W. Bogaerts and L. Chrostowski, "Silicon Photonics Circuit Design: Methods, Tools and Challenges," Laser Photon. Rev., vol. 1700237, pp. $1-29,2018$.

[58] D. Thomson, A. Zilkie et al., "Roadmap on silicon photonics," J. Opt., vol. 18, no. 7, p. $073003,2016$.

[59] M. Liu, X. Yin et al., "A graphene-based broadband optical modulator," Nature, vol. 474, no. 7349, pp. 64-67, 2011.

[60] J. Faneca, T. Perova, V. Tolmachev, and A. Baldycheva, "Onedimensional multi-channel photonic crystal resonators based on SiliconOn-Insulator with high quality factor," Front. Phys., vol. 6, 2018.

[61] W. Heni, C. Hoessbacher et al., "High speed plasmonic modulator array enabling dense optical interconnect solutions," Opt. Express, vol. 23, no. 23, p. 29746, 2015.

[62] L. Alloatti, R. Palmer et al., "100 GHz silicon-organic hybrid modulator," Light Sci. Appl, vol. 3, pp. 5-8, 2014.

[63] C. Koos, P. Vorreau et al., "All-optical high-speed signal processing with silicon-organic hybrid slot waveguides," Nat. Photonics, vol. 3, no. 4 pp. 216-219, 2009.

[64] C. G. Littlejohns, M. Nedeljkovic et al., "Next Generation Device Grade Silicon-Germanium on Insulator," Sci. Rep., vol. 5, 2015.

[65] C. Ríos, M. Stegmaier et al., "Integrated all-photonic non-volatile multilevel memory," Nat. Photonics, vol. 9, no. 11, pp. 725-732, 2015.

[66] C. Rios, P. Hosseini, C. D. Wright, H. Bhaskaran, and W. H. P. Pernice, "On-chip photonic memory elements employing phase-change materials," Adv. Mater., vol. 26, no. 9, pp. 1372-1377, 2014.

[67] C. Rios, M. Stegmaier et al., "Controlled switching of phase-change materials by evanescent-field coupling in integrated photonics [Invited]," Opt. Mater. Express, vol. 8, no. 9, p. 2455, 2018.

[68] M. Wuttig, H. Bhaskaran, and T. Taubner, "Phase-change materials for non-volatile photonic applications," Nat. Photonics, vol. 11, no. 8, pp. 465-476, 2017.

[69] M. Wuttig and N. Yamada, "Phase-change materials for rewriteable data storage," Nat. Mater, vol. 6, p. 824, 2007.

[70] J. Hegedüs and S. R. Elliott, "Microscopic origin of the fast crystallization ability of Ge-Sb-Te phase-change memorymaterials," Nat. Mater., vol. 7, no. 5, pp. 399-405, 2008.

[71] T. Matsunaga and N. Yamada, "A study of highly symmetrical crystal structures, commonly seen in high-speed phase-change materials, using synchrotron radiation," Jpn. J. Appl. Phys. Pt. 1, vol. 41, no. 3B, pp. 1674-1678, 2002.

[72] C. Ríos, N. Youngblood et al., "In-memory computing on a photonic platform," Sci. Adv., vol. 5, no. 2, 2019.

[73] C. R. de Galarreta, A. M. Alexeev et al., "Nonvolatile Reconfigurable Phase-Change Metadevices for Beam Steering in the Near Infrared," Adv. Funct. Mater, vol. 28, no. 10, 2018.

[74] J. Zheng, A. Khanolkar et al., "Gst-on-silicon hybrid nanophotonic integrated circuits: a non-volatile quasi-continuously reprogrammable platform," Opt. Mater. Express, vol. 8, no. 6, pp. 1551-1561, Jun 2018.

[75] H. Zhang, L. Zhou et al., "Ultracompact si-gst hybrid waveguides for nonvolatile light wave manipulation," IEEE Photonics J., vol. 10, no. 1, pp. 1-10, Feb 2018.

[76] R. Soref, "Enabling $2 \mu \mathrm{m}$ communications," Nat. Photonics, vol. 9, p. $358,2015$.

[77] P. J. Roberts, F. Couny et al., "Ultimate low loss of hollow-core photonic crystal fibres," Opt. Express, vol. 13, no. 1, pp. 236-244, 2005. 
[78] E. Desurvire, C. Kazmierski et al., "Science and technology challenges in XXIst century optical communications," C. R. Phys., vol. 12, no. 4, pp. $387-416,2011$.

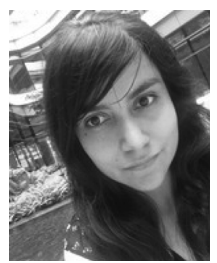

Thalía Domínguez Bucio Dr. Thalía Doínguez Bucio is a research fellow in the Silicon Photonics group at the Optoelectronics Research Centre (ORC) in the University of Southampton. She received her B. S degree in electronic and computer engineering from the Monterrey Institute of Technology (Mexico, 2012) and her M.Sc. in photonic technologies from the University of Southampton (UK, 2013). She received her PhD in optoelectronics from the ORC in 2018. Her research interests lie in the development of new material platforms for photonic.

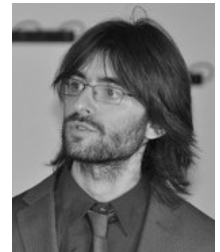

Cosimo Lacava Dr. Cosimo Lacava is a Senior Research Fellow in the ORC. He obtained his $\mathrm{PhD}$ in Optoelectronics and Electronic Engineering in 2014. He continued working at the Quantum Electronics Laboratory as Postdoc researcher, working on the development of novel Silicon Photonics transceiver device for the next generation optical networks. At the end of 2014 he moved to Southampton to join ORC. His research interests are: nonlinear optics, all optical signal processing, silicon photonic devices and integrated optics.

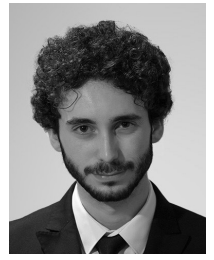

Marco Clementi Marco Clementi is a $\mathrm{PhD}$ student in Physics in the Photonics and Nanostructures research group at the University of Pavia, Italy. He graduated with honours in Electronic Engineering at the University of Pavia in 2016, with a thesis on optical quantum information processing. His research interests include photonics and integrated nonlinear optics with particular focus on photonic crystal cavities.

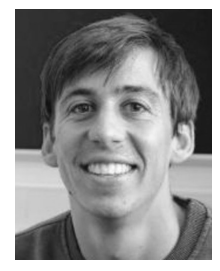

Joaquin Faneca Joaquin Faneca is a $\mathrm{PhD}$ student at the University of Exeter in the CDT Metamaterials. He received his B.S degree in Physics from the Universidad de Sevilla (Spain,2015) and his M.Sc in Novel photonics and electronics technologies from Universidad Complutense de Madrid (Spain,2016). His research interests lies in the development of a reconfigurable photonic integrated platform based on smart materials for optical communication applications.

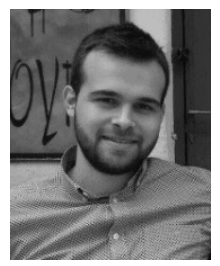

Ilias Skandalos Ilias Skandalos is a $\mathrm{PhD}$ student in the Silicon Photonics Group at the Optoelectronics Research Center (ORC) of University of Southampton. He received his integrated $\mathrm{BSc}$ and $\mathrm{MSc}$ degree in Electrical and Computer Engineering from Aristotle University of Thessaloniki (2017). His research interests lie in the field of optical modulators, exploiting electro-absorption effects using various platforms, towards the realisation of efficient optical interconnects.

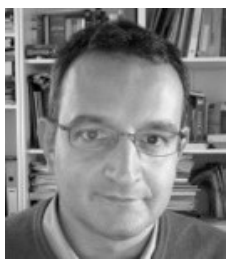

Matteo Galli Dr. Matteo Galli is assistant professor at the Physics Department of the University of Pavia, where he leads the Laboratory of Integrated Quantum Photonics. His research interests span several topics in solidstate physics, among which radiation-matter interaction in photonic nanostructures, light emission in silicon, integrated nonlinear and quantum photonics, plasmonic nanostructures for biosensing and photovoltaics. He actively contributed in these fields by introducing several original experimental techniques and methods, such as angle-resolved ATR micro-spectroscopy, white-light interferometry and resonant scattering of light. Overall, he is co-author of more than 100 journal publications and two patents.

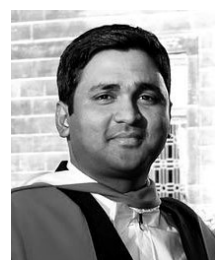

Kapil Debnath Dr. Debnath is an Assistant Professor at the Electronics and Electrical Engineering Department of IIT Kharagpur. He completed his BTech in Electronics and Telecommunication Engineering from NIT Silchar in 2004 and MTech in Applied Optics from IIT Delhi in 2009. He carried out his $\mathrm{PhD}$ research at the University of St Andrews under the guidance of Prof. Thomas Krauss from 2009-2013. During his PhD, he developed a novel optical interconnect architecture based on Photonic Crystal Cavities suitable for both on-chip and off-chip communications. Using this technology, he demonstrated electro-optic modulators with worlds lowest switching energy. In 2013, his patented architecture resulted in a proof-ofconcept project funded by Scottish Enterprise. In 2015, he moved to the University of Southampton and worked as a postdoctoral research fellow with Prof. Graham Reed and Prof. Shinichi Saito. At the University of Southampton, he developed a unique fabrication process and demonstrated a capacitive silicon modulator with speed up to $25 \mathrm{Gbps}$. His current research interest is in the fields of attojoule optoelectronic components for on-chip communication, silicon solar cells, integrated nonlinear photonics.

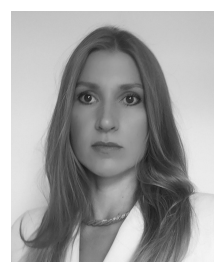

Anna Baldycheva Dr. Anna Baldycheva received the B.Sc. degree with Honors from Physics Faculty at St. Petersburg State University. In 2008, she was awarded prestigious research fellowship from ICGEE (Irish Research Council) to pursue the Ph.D. in Nano and Micro Si Photonics at the University of Dublin, Trinity College. During her $\mathrm{PhD}$ she lead an independent research project at Tyndall National Institute with a grant funded by Science Foundation Ireland. After completion of her PhD in 2012, she joined MIT as a Post-Doc with Prof Mike Watts. In November 2014 she moved to UK to take on a position as an assistant professor at University of Exeter. Dr. Baldycheva has published over 50 peer-reviewed papers and conference proceedings. She is also a member of SPIE, Women in Optics, Optical Society of America (OSA), European Microscopy Society (EMS), Royal Microsopy Society (RMS) and Microscopy Society of Ireland (MSI). Prof Baldycheva is an associate editor of the Nature Scientific Reports and is on the board of the Royal Microscopy Society Engineering.

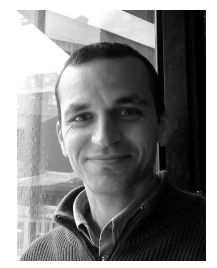

Periklis Petropoulos Dr. Periklis Petropoulos graduated from the Department of Electrical Engineering and Information Technology, University of Patras, Greece in 1995. He received the MSc degree in Communications Engineering from the University of Manchester Institute of Science and Technology, UK and the PhD degree in Optical Telecommunications from the Optoelectronics Research Centre (ORC), University of Southampton, UK, where he currently works as a Professor. His research interests lie in the fields of optical communications, all-optical signal processing and novel fibre and waveguide technologies. He has participated in several European Union and national research projects in the field of optical communications. His research has produced more than 470 papers in technical journals and conference proceedings, including several invited and postdeadline papers in major international conferences, and holds 6 patents. Dr. Petropoulos has served as member of the Technical Programme Committees for several international conferences, including the European Conference on Optical Communication (ECOC), the Optical Fiber Communication (OFC) conference and the European Conference on Lasers and Electro-Optics (CLEO/Europe). He is a Fellow of the Optical Society of America.

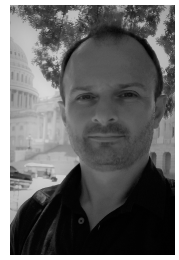

Frederic Gardes Dr Frederic Gardes is an associate professor at the Optoelectronic Research centre at the university of Southampton in the UK. Dr Gardes received his $\mathrm{PhD}$ in silicon photonics under the supervision of Professor Graham Reed. His research background covers CMOS photonics and in particular high-speed active optical devices in silicon, germanium and mid index waveguide. In 2005, he initiated work on silicon optical depletion modulators and was the first to predict optical modulators operating above $40 \mathrm{GHz}$. These pn junctions based modulators were demonstrated from(2008) in ring resonators and in MZI in 2010, and 2011 at data rates up $50 \mathrm{~Gb} / \mathrm{s}$. The most recent work is the focus activity of Dr Gardes research group with activity in SiGe epitaxy on insulator for modulation, detection and light emission and multilayer waveguiding material systems such as silicon nitride. Dr Gardes has authored more than 250 publications in the field of silicon photonics and has multiple patents. Dr Gardes is also a member of several international conference programme committees. 